\title{
A Review of Advancements in Nasal Airway Measurement
}

\author{
Katharine Hamlett, and B Nirmal Kumar* \\ Department of Otolaryngology, Royal Albert Edward Infimary, Wigan, WN1 2NN, UK
}

*Corresponding author: B Nirmal Kumar, Department of Otolaryngology, Royal Albert Edward Infimary, Wigan, WN1 2NN, UK, E-mail: nirmalkumar@doctors.org.uk

Received: 30 Sep, 2019 | Accepted: 21 Oct, 2019 | Published: 25 Oct, 2019

Citation: Hamlett K, Kumar BN (2019) A Review of Advancements in Nasal Airway Measurement. J Surg Open Access 5(5): dx.doi. org/10.16966/2470-0991.196

Copyright: (C) 2019 Hamlett K, et al. This is an open-access article distributed under the terms of the Creative Commons Attribution License, which permits unrestricted use, distribution, and reproduction in any medium, provided the original author and source are credited.

\begin{abstract}
Medical therapies and operative techniques for intra-nasal disease are constantly evolving; it is therefore imperative that outcomes from treatments and operative techniques can be objectively measured to ensure surgical efficacy. Investigations that measure the nasal airway can help to guide the management of sino-nasal diseases, which can subsequently have a significant impact on patients' quality of life. Subjective methods of measurement include; anterior rhinoscopy, rigid nasendoscopy, VAS score and SNOT-22. Objective measures include; nasal peak flow testing, acoustic rhinometry, anterior/posterior rhinomanometry and imaging studies. Unfortunately, many objective measurement techniques correlate poorly with patients' perceptions of disease severity, which questions the clinical relevance of these techniques. Robust, objective methods for measuring the nasal airway may help to guide future treatments, and should ideally correlate with patients' symptoms to truly tackle the burden of nasal disease.
\end{abstract}

Keywords: Acoustic rhinometry; Airway measurement; Nasal airway; nasal patency; Peak flow; Rhinomanometry; Rhinosinusitis

\section{Introduction}

As the nasal cavity is essentially the gateway to the rest of the airway and the nasal mucosa is the first point of contact for air entering the respiratory tract, the nasal cavity plays a vital role in the homeostasis of respiration [1]. The nasal cavity humidifies and warms the inhaled air entering the respiratory tract, drains the paranasal sinuses and lacrimal ducts, filters particles and pathogens in the inhaled air, contributes to our innate and adaptive immunity and is the organ responsible for olfaction $[1,2]$.

Investigations that measure the nasal airway can help to guide the management of nasal diseases, which can have a significant impact on patients' quality of life [2]. Nasal obstruction is one of the most prevalent symptoms in patients presenting to Otolaryngology outpatient clinics [3]. Subjective methods of measuring the nasal airway include methods such as; anterior rhinoscopy and rigid nasendoscopy. These techniques allow the clinician to directly visualise the nasal cavity, make a visual assessment of nasal patency and help to identify pathology. However, these techniques do not provide any objective measurement of the nasal cavity, which can be vital when assessing disease progression and treatment outcomes, as objective measurement is often considered fundamental for evidencebased medical practice [4].

Objective techniques for measuring the nasal airway include; nasal peak flow testing, acoustic rhinometry, anterior and posterior rhinomanometry and imaging studies. Objective tests of the nasal airway must be tolerable for patients, easily reproducible, accurate, standardised and safe $[5,6]$. Unfortunately, many objective measurement techniques correlate poorly with patients' perceptions of disease severity [4]. This begs the question; how useful are these tests in measuring the nasal airway and subsequent treatment outcomes? Furthermore, this also highlights the need to develop novel tests that truly reflect patient symptoms related to pathology within the nasal cavity.

This evidence-based narrative review article highlights a medical field in which we still have much to understand. Furthermore, a significant proportion of otolaryngology referrals are comprised of nasal complaints, with many requiring long-term medical treatment and surgical interventions. Accurate, objective assessment of the nasal airway may potentially help to reduce the burden of disease for patients, by allowing for more effective assessment of treatment response and a reduction in unnecessary surgical procedures. Therefore, this article introduces the pathophysiology and an outline of the various techniques for measuring the nasal airway, including an overview of the evidence for the latest advancements.

\section{Overview: Nasal Airway Pathophysiology}

Olfaction is dependent on the eddies and turbulence created by the nasal anatomy [1]. The anatomical configuration means that an only a minor amount of congestion and tissue swelling can lead to considerable changes in nasal patency and airflow [1]. This is described by Poiseuille's law, whereby flow is directly proportional to the radius and the pressure difference within and inversely proportional to length and viscosity of the liquid within [7]. Therefore, only minor changes 
in the radius of the nasal airway can lead to a much greater reduction in nasal airflow, due to increased nasal airway resistance. This can in turn have a considerable impact on the symptoms experienced by patients. It is due to a combination of these factors that patients with conditions such as rhinosinusitis struggle with symptoms such as nasal obstruction and congestion.

The narrowest part of the normal nasal airway, and considered a zone of high airway resistance, is the nasal valve. There are in fact, both internal and external nasal valves [8]. The external nasal valve is an area of the nasal vestibule delimited by the alar rim, nasal sill and caudal septum [9]. The internal nasal valve is delimited by the nasal septum, the caudal margins of the upper lateral cartilage and the anterior portion of the inferior nasal turbinate [10]. The external nasal valve supposedly contributes to approximately $50 \%$ of the total resistance to airflow within the whole respiratory tract [11]. Many factors can affect nasal valve patency such as; trauma, perioperative damage, ageing and inflammatory conditions. Nasal airway resistance can be also affected by numerous other physiological and pathological processes.

\section{Measurement Techniques}

There are several measurement techniques for quantifying nasal airway obstruction and nasal airway disease that include both subjective and objective measurement techniques (Table 1).

\section{Subjective techniques}

Bedside examination and cottle manoeuvre: Visual, external inspection of the nose is usually the preliminary bedside examination made when performing any form of examination of the nose. It often provides clinicians with instantaneous information about the potential patency of the nasal airway, based on the anatomical appearance of the nose, identification of external abnormalities and the appearances during inspiration and expiration [2]. The traditional cottle manoeuvre involves an assessment of subjective nasal patency during nasal inspiration both before and after the ipsilateral cheek is pulled laterally, to open the external nasal valve. It has been previously used to evaluate post-surgical outcomes [8]. However, a recent cohort study has shown that the cottle manoeuvre has limited clinical utility in predicting surgical outcomes [12]. This limits its usefulness in the clinical setting, and suggests we should not rely on the cottle manoeuvre as a definitive indicator of nasal valve disease.

Anterior rhinoscopy/flexible nasendoscopy: Anterior rhinoscopy provides a simple, readily available, cheap technique for observing the anterior aspect of the cavum nasi. It allows the clinician to make a quick, subjective assessment of the nasal cavity, with appreciation of the nasal valve and any obvious obstructions to the anterior nasal cavities [2]. Anterior rhinoscopy involves inspection of the anterior

Table 1: Subjective and objective measures of the nasal airway.

\begin{tabular}{|l|l|}
\hline \multicolumn{1}{|c|}{ Subjective Measures } & \multicolumn{1}{c|}{ Objective Measures } \\
\hline Nasal Inspection & Peak Nasal Inspiratory Flow (PNIF) \\
\hline Cottle Manouevre & Peak Nasal Expiratory Flow (PNEF) \\
\hline Anterior Rhinoscopy & Anterior Rhinomanometry \\
\hline Nasendoscopy (Rigid/Flexible) & Posterior Rhinomanometry \\
\hline NOSE Scale & Acoustic Rhinometry \\
\hline SNOT-22 Score & Computational Streamline Rhinometry \\
\hline Visual Analog Scale (VAS) & Computed Tomography (CT) Imaging \\
\hline & Magnetic Resonance Imaging (MRI) \\
\hline
\end{tabular}

part of the nasal cavity using a thudicum nasal speculum to open the nostrils. This test is limited by the extent of views obtained of the nasal airway, and does not provide any objective measurement of the nasal airway. It does however provide a good diagnostic tool to help identify many of the common intranasal pathologies. The use of a metal spatula or lacks tongue depressor beneath the nostrils allows for a quick, crude, bedside assessment of nasal patency, whereby the clinician observes for bilateral misting on the cold metal. Rigid and flexible nasendoscopy both offer more comprehensive view of the nasal passages, with better appreciation of intranasal disease. A direct visual assessment of the volume and patency of the nasal airway can be made, although this is subjective. If polyps are present, nasal endoscopy allows clinicians to grade the level of obstruction, although good correlation between polyp size and perception of congestion has not yet been found [2].

NOSE scale: The Nasal Obstruction Symptom Evaluation (NOSE) scale was developed as a quality of life measure for patients with nasal obstruction, and was validated as a subjective measure [13]. The NOSE scale consists of simple, easily administered quality of life questions based around patient perceptions of nasal patency and congestion, and can be used in the evaluation of treatment outcomes [14]. A systematic review on advances in diagnostic techniques for nasal airway obstruction showed that the NOSE scale had good correlation with other subjective nasal airway measures, although revealed inconsistent correlation with objective measures [15].

SNOT-22: The modified Sino-Nasal Outcome Test (SNOT-22) is a 22-question symptom score adopted for patients with sino-nasal symptoms [16]. Studies on the correlation of the SNOT-22 and objective measure of nasal airway obstruction score and have shown differing results, with some demonstrating statistically significant relationships, whilst others reveal contrasting results [15]. The SNOT-22 has been shown to be an effective measure of the impact of chronic rhinosinusitis symptoms on patients' quality of life, while also providing an outcome measure following surgical intervention [16].

Visual analogue scale: The Visual Analog Scale (VAS) has previously been used to subjectively quantify patient perception of nasal patency [17]. The patient is directed to cover one nostril, whilst rating their ability to breathe through the contralateral nostril on a scale from one (no obstruction) to 10 (severe obstruction). VAS is a unilateral measure of patency, contrasting to the NOSE scale, which is a bilateral measure. It is a simple and reliable measure of nasal patency that can easily be performed within a clinic setting.

\section{Objective techniques}

Nasal patency is a measure of how open the nasal passages are, and is usually quantified by nasal cross-sectional area or volume [18]. Nasal patency is a prerequisite for the efficient transport of inspired air to the lungs. Objective measures of nasal patency include techniques to measure nasal cross-sectional diameter, nasal airflow and nasal airway resistance [2].

Peak nasal inspiratory/expiratory flow: Nasal airflow during respiration can be measured, focusing specifically on Peak Nasal Inspiratory Flow (PNIF) (Figure 1) and Peak Nasal Expiratory Flow (PNEF). These techniques have arisen in a similar fashion to that of peak flow testing for respiratory disease. PNIF is performed by placing the mask over the nose, and performing a single quick inspiration, measuring the peak inspiratory airflow in both nostrils simultaneously. PNIF is a more commonly used and validated test, and avoids issues such as mucous deposition in the peak flow meter following PNEF 


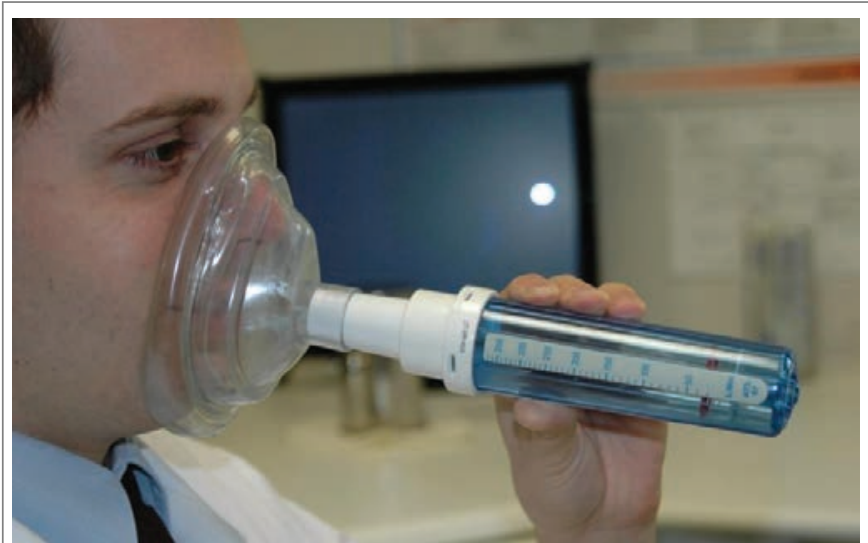

Figure 1: Peak nasal inspiratory flow meter technique (Scadding G, et al. [2]).

testing. PNIF is a patient-dependent test, and therefore cannot be used when there might be a financial incentive for patients with a suspected occupational disease [19]. Phagoo SB, et al. [5] describes that nasal peak flow results can be affected by intra-pulmonary airway obstruction, such as in patients with rhinitis with concurrent asthma. When comparing PNIF and PNEF, PNIF is considered a superior test of nasal patency. When comparing maximal PNIF and PNEF before and after mucosal vasoconstriction with an adrenergic agonist, studies have shown that there is a greater overall increase in PNEF compared to PNIF [5]. Numerous studies have previously demonstrated the effectiveness of using PNIF to monitor pre- and post-treatment changes. PNIF is useful to detect small changes in nasal patency, including in the context of nasal provocation testing [6]. PNIF has been found to be a cheap, reliable and reproducible test which provides a direct measure of nasal airflow, showing statistically significant improvements in nasal airflow following functional septorhinoplasty [20].

Rhinomanometry: Airflow and pressure during nasal respiration can be measured by anterior and posterior rhinomanometry. Anterior rhinomanometry involves placement of a pressure probe in one nostril, although the contralateral nostril must be patent to allow measurement [2]. This test provides an objective measure of nasal airways resistance [19]. Yaniv E, et al. [3] conducted a study looking at patients pre- and post-Functional Endoscopic Sinus Surgery (FESS), and measured the nasal airway using rhinomanometry and a subjective visual scale on perception of nasal obstruction. They hypothesised that rhinomanometry values post-surgery would correlate poorly with the subjective perception of nasal airflow. This was attributed to the fact that post-uncinectomy, patients experience a significant improvement in nasal airflow, however the uncinate is such a small nasal projection, it does very little to change airflow or resistance, and therefore is unlikely to affect rhinomanometry values. The study proved their hypothesis correct; there was little change in rhinomanometry results. The benefits of rhinomanometry, is that it provides objective data on nasal resistance, assessing each nostril individually, it is quick to perform and a relatively simple technique. Conversely, the equipment can be costly, not easily portable, requires an operator and cannot be performed in unilateral complete obstruction of the nasal passage [2].

Acoustic rhinometry: The volume of the nasal cavity can also be measured using acoustic rhinometry. Acoustic rhinometry has been described as a quick, reliable and reproducible method for measuring nasal volume. It involves an acoustic sound wave emission from a probe place in the nasal passages one at a time during a breath hold, measuring the reflection of the sound wave returning to the measurement probe (Figure 2), and using mathematical algorithms to calculate the nasal cavity volume [2]. This delineates the nasal cavity volume and is safe and simple to use. Drawbacks include the fact this technique is not physiological, as it is performed during a breathhold and it may be affected by the nasal cycle. Acoustic rhinometry overestimates nasal volume due to leakage of sound to the sinuses [21]. In a study comparing acoustic rhinometry, subjective symptoms and endoscopic findings in paediatric patients with nasal airway obstruction, endoscopic rhinitis score on nasal endoscopy was the only significant predictor of the VAS. Acoustic rhinometry was not a reliable predictor [22].

Imaging modalities: Varied imaging techniques may be used to evaluate the nasal airway. CT and Magnetic Resonance Imaging (MRI) can be used to measure the cross-sectional area of the nasal passages, and are often used in pre-operative planning and for assessment of intra-nasal disease. These techniques can also help with validation of other measurement techniques. However, there are understandable cost and time implications involved with both methods, with the addition of radiation exposure in CT imaging [18]. Both CT and MRI can be used to form 3D models of the nasal airway, to aid in understanding the factors affecting the nasal airway, although they are clearly not quick, economical, bedside tests.

In a study investigating the relationship between Minimal CrossSectional Area (mCSA) on imaging and nasal resistance to airflow the authors hypothesised that there is a strong correlation between nasal resistance and mCSA, but only for severe constrictions where the mCSA is below a Critical Area (critA) [21]. Using Computed Tomography (CT) imaging they developed models of the nasal anatomy in patients' pre and post-nasal obstruction surgery. Using steady-state laminar airflow simulations, cross-sectional areas of the nasal cavity were mapped, with the technique named 'Computational Streamline Rhinometry'. This study, although only performed on a small study size, found that nasal resistance was strongly related to mCSA, but only when the mCSA was less than the critA. This finding provides theoretical reasoning for the apparent poor correlation between subjective nasal patency and mCSA [21]. Other similar studies have agreed with this conclusion [17]. It suggests that there are several factors contributing to nasal patency, contributing to the enigmatic relationship between rhinological form and function.

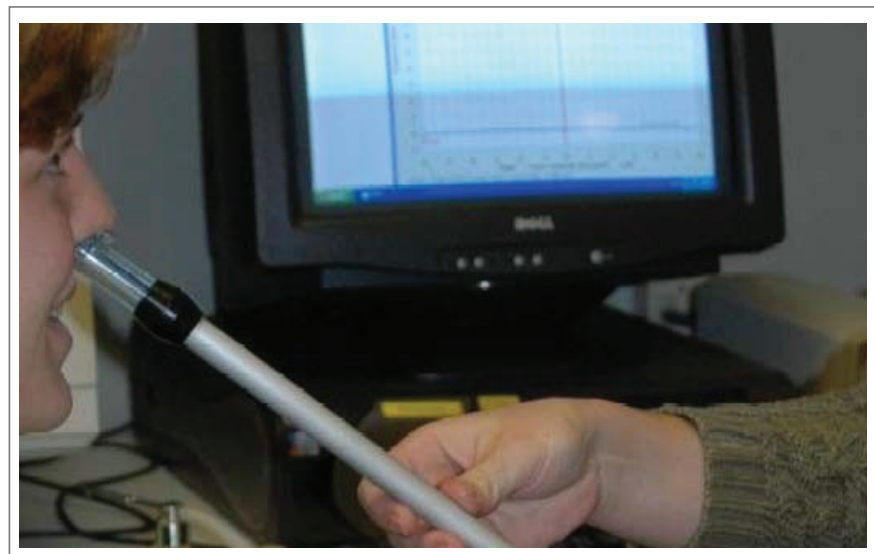

Figure 2: Acoustic rhinometry probe and technique. (Scadding G, et al. [2]). 


\section{Review Limitations}

To reduce bias, the primary author of this article widely searched online databases available on nasal airway measurement to synthesize the literature for the review and then further hand-searched any relevant article's references. However, whilst this article covers a wide breadth of literature, it is a narrative review, and therefore lacks in the systematic amalgamation of the current evidence base that can help to minimise bias. Perhaps this highlights an area for future study, which may encourage deeper comprehension of nasal airway measurement techniques. Unfortunately, despite the literature available, there remains little consensus on the optimum measurement tools for the nasal airway which impacts on the clinical interpretation of study findings.

\section{Future Advances}

There are limited novel techniques presently being designed to assess the nasal airway, although several clinical trials are underway which may broaden our knowledge base. An ongoing US study listed on the ClinicalTrials.gov database intends to evaluate the use of thermal imaging as a diagnostic tool for assessing nasal airflow [23]. In their study protocol, they hypothesise that a reduction in the patency of the nasal airway, and therefore a reduction in airflow, may lead to loss of the physiological cooling mechanisms that occur during the nasal respiratory cycle, and this may be possible to identify via thermal imaging. Time will tell as to whether this technique will contribute to our future clinical practice. The NAIROS trial is an ongoing randomised controlled trial taking place between October 2017 and May 2020 [24]. Patients are recruited with nasal obstruction and concurrent septal deviation, with random allocation of participants into two groups: Septal surgery with reduction of the inferior turbinates versus medical therapy with saline nasal spray and Mometasone furoate nasal spray for 6 months' duration. The clinical outcomes will be measured both subjectively and objectively via measures such as the NOSE scale, SNOT-22 score and PNIF. These results may contribute to further comprehension of techniques of nasal airway measurement, and impact on future clinical practice.

\section{Conclusion}

Thousands of patients undergo endoscopic sinus surgery, septoplasty and other rhinological procedures every day worldwide, with many patients having relative indications for surgery such as poor symptom control and persistent nasal disease [3,25]. Medical therapies and operative techniques for intra-nasal disease are constantly evolving; it is therefore imperative that outcomes from these operative techniques can be objectively measured to ensure efficacy. Robust, objective methods for measuring the nasal airway may help to guide future treatments, and should ideally correlate with patients' symptoms to truly tackle the burden of nasal disease [4]. Many objective measures of the nasal airway have been shown to correlate poorly to subjective perceptions of nasal airway disease, adding to the challenge of discovering a measure that truly reflects intra-nasal form and function.

\section{Acknowledgements}

No acknowledgements.

\section{Conflicts of Interest}

Author declares no conflicts of interest.

\section{Funding}

Nil sources of funding obtained.

\section{References}

1. Hilberg $\mathrm{O}$ (2002) Objective measurement of nasal airway dimensions using acoustic rhinometry: methodological and clinical aspects. Allergy 57: 5-39.

2. Scadding G, Hellings P, Alobid I, Bachert C, Fokkens W, et al. (2011) Diagnostic tools in Rhinology EAACl position paper. Clin Transl Allergy 1: 2.

3. Yaniv E, Hadar T, Shvero J, Raveh E (1997) Objective and subjective nasal airflow. Am J Otolaryngol 18: 29-32.

4. Rhee JS, Book DT, Burzynski M, Smith TL (2003) Quality of Life Assessment in Nasal Airway Obstruction. Laryngoscope 113: 11181122.

5. Phagoo SB, Watson RA, Pride NB (1997) Use of nasal peak flow to assess nasal patency. Allergy 52: 901-918.

6. Teixeira RU, Zappelini CE, Alves FS, da Costa EA (2011) Peak nasal inspiratory flow evaluation as an objective method of measuring nasal airflow. Braz J Otorhinolaryngol 77: 473-480.

7. Bloching MB (2007) Disorders of the nasal valve area. GMS Curr Top Otorhinolaryngol Head Neck Surg 6: 1-13.

8. Fung E, Hong P, Moore C, Taylor SM (2014) The effectiveness of modified cottle maneuver in predicting outcomes in functional rhinoplasty. Plast Surg Int 2014: 618313.

9. Constantian MB (1994) The incompetent external nasal valve: pathophysiology and treatment in primary and secondary rhinoplasty. Plast Reconstr Surg 93: 919-931.

10. Rhee JS, Weaver EM, Park SS, Baker SR, Hilger PA, et al. (2010) Clinical consensus statement: Diagnosis and management of nasal valve compromise. Otolaryngol Head Neck Surg 143: 48-59.

11. Dahl R, Mygind N (1998) Anatomy, physiology and function of the nasal cavities in health and disease. Adv Drug Deliv Rev 29: 3-12.

12. Bonaparte JP, Campbell R (2018) A prospective cohort study assessing the clinical utility of the Cottle maneuver in nasal septal surgery. J Otolaryngol Head Neck Surg 47: 45.

13. Stewart MG, Witsell DL, Smith TL, Weaver EM, Yueh B, et al. (2004) Development and Validation of the Nasal Obstruction Symptom Evaluation (NOSE) Scale. Otolaryngol Head Neck Surg 130: 157-163.

14. Lipan MJ, Most SP (2013) Development of a Severity Classification System for Subjective Nasal Obstruction. JAMA Facial Plast Surg 15: 358-361.

15. Mohan S, Fuller JC, Ford SF, Lindsay RW (2018) Diagnostic and Therapeutic Management of Nasal Airway Obstruction: Advances in Diagnosis and Treatment. JAMA Facial Plast Surg 20: 409-418.

16. Hopkins C, Gillett S, Slack R, Lund VJ, Browne JP (2009) Psychometric validity of the 22-item Sinonasal Outcome Test. Clin Otolaryngol 34: 447-454.

17. Casey KP, Borojeni AA, Koenig LJ, Rhee JS, Garcia GJ (2017) Correlation between Subjective Nasal Patency and Intranasal Airflow Distribution. Otolaryngol Head Neck Surg 156: 741-750.

18. Ottaviano G, Fokkens WJ (2016) Measurements of nasal airflow and patency: a critical review with emphasis on the use of peak nasal inspiratory flow in daily practice. Allergy 71: 162-174.

19. Pirilä T, Nuutinen J (2001) Acoustic rhinometry, rhinomanometry and the amount of nasal secretion in the clinical monitoring of the nasal provocation test. Clin Exp Allergy 28: 468-477. 
20. Fuller JC, Gadkaree SK, Levesque PA, Lindsay RW (2019) Peak nasal inspiratory flow is a useful measure of nasal airflow in functional septorhinoplasty. Laryngoscope 129: 594-601.

21. Garcia GJM, Hariri BM, Patel RG, Rhee JS (2016) The relationship between nasal resistance to airflow and the airspace minimal crosssectional area. J Biomech 49: 1670-1678.

22. Isaac A, Major M, Witmans M, Alrajhi Y, Flores-Mir C, et al. (2015) Correlations between acoustic rhinometry, subjective symptoms, and endoscopic findings in symptomatic children with nasal obstruction. JAMA Otolaryngol Head Neck Surg 141: 550-555.

23. Clinical Trials gov (2018) Thermal imaging as a potential diagnostic tool of nasal airflow.

24. Rennie (2018) Nasal Airway Obstruction Study-NAIROS.

25. Pynnonen MA, Davis MM (2014) Extent of sinus surgery, 2000 to 2009: a population-based study. Laryngoscope 124: 820-825. 